\title{
A NEW NORMAL FORM FOR QUARTIC EQUATIONS*
}

\author{
BY RAYMOND GARVER
}

1. Introduction. There are a number of well known normal forms to which the general quartic equation may be reduced by means of a Tschirnhaus transformation, without requiring the solution of any equation of higher than the third degree. The reduction to $y^{4}+c_{3} y+c_{4}=0$ or to $y^{4}+c_{2} y^{2}+c_{4}=0$ requires no comment. Bring first showed how to obtain the form $y^{4}+c_{1} y^{3}+c_{4}=0$, by applying a simple reciprocal transformation (which is reducible to a Tschirnhaus transformation) to the first form above. $\dagger$ The reduction to the binomial form $y^{4}+c_{4}=0$ by an ordinary third degree transformation leads to a sixth degree equation, but Lagrange was able to show (by an a priori proof) that this sextic will factor into three quadratics whose coefficients are themselves roots of cubic equations. $\ddagger$ The required factorization would, however, be difficult actually to obtain; the necessary transformation may be obtained more conveniently in another way. $\$$

I wish in this paper to discuss a new normal form, $y^{4}+c_{2} y^{2}$ $=0$. I first give an a priori proof, based on that of Lagrange for the transformation to binomial form, that the reduction does not involve the solution of any equation of higher than the third degree. Then, using a theorem of Hermite and certain results of Cayley, I consider the setting up of the necessary transformation.

2. The a Priori Proof. If we apply the transformation

$$
y=x^{3}+k_{2} x^{2}+k_{3} x+k_{4}
$$

to the general quartic equation

* Presented to the Society, April 7, 1928.

$\dagger$ Bring's work was published in 1786. It is reproduced in Grunert's Archiv, vol. 41 (1864), pp. 105-112.

$\ddagger$ See his Oeuvres, vol. III, Paris, 1869 , pp. $284-295$. His result was originally published in $\mathbf{1 7 7 0 .}$

$\S$ See this Bulletin, vol. 33 (1927), No. 6. 


$$
x^{4}+a_{1} x^{3}+a_{2} x^{2}+a_{3} x+a_{4}=0,
$$

the conditions that the transformed equation in $y$ lack its second, fourth and fifth terms are, as can easily be verified by making use of Newton's relations,

$$
\sum y=0, \sum y^{3}=0,\left(\sum y^{2}\right)^{2}-2 \sum y^{4}=0 .
$$

But the first of these equations is linear in $k_{2}, k_{3}, k_{4}$, the second is of the third degree, while the third is of the fourth degree. Hence if we eliminate two of the $k_{i}$, say $k_{3}$ and $k_{4}$, between these equations, we shall expect to be led to a final equation of the twelf th degree in $k_{2}$.

The problem is to show that $k_{2}$ can be found without requiring the solution of any equation of higher than the third degree. To do this, it is necessary to express $k_{2}$ as a function of the roots $x_{1}, x_{2}, x_{3}, x_{4}$ of the given quartic, on the assumption that the transformed equation is $y^{4}+c_{2} y^{2}=0$. The roots of this latter equation are 0,0 , and, let us say, $r$ and $-r$. We must then have, from (1),

$$
\left\{\begin{aligned}
0 & =x_{1}{ }^{3}+k_{2} x_{1}{ }^{2}+k_{3} x_{1}+k_{4}, \\
0 & =x_{2}{ }^{3}+k_{2} x_{2}{ }^{2}+k_{3} x_{2}+k_{4}, \\
r & =x_{3}{ }^{3}+k_{2} x_{3}{ }^{2}+k_{3} x_{3}+k_{4}, \\
-r & =x_{4}{ }^{3}+k_{2} x_{4}{ }^{2}+k_{3} x_{4}+k_{4} .
\end{aligned}\right.
$$

It is now a matter of simple algebra to solve these equations for $k_{2}$; the result may be written in the form

$$
k_{2}=-\frac{\left(x_{1}^{3}+x_{2}^{3}-x_{3}^{3}-x_{4}^{3}\right)\left(x_{1}-x_{2}\right)-\left(x_{1}^{3}-x_{2}^{3}\right)\left(x_{1}+x_{2}-x_{3}-x_{4}\right)}{\left(x_{1}^{2}+x_{2}^{2}-x_{3}^{2}-x_{4}^{2}\right)\left(x_{1}-x_{2}\right)-\left(x_{1}^{2}-x_{2}^{2}\right)\left(x_{1}+x_{2}-x_{3}-x_{4}\right)} \text {. }
$$

We may now find the number of different values which $k_{2}$ can assume by making the 24 possible permutations on the roots $x_{1}, x_{2}, x_{3}, x_{4}$. If the reader goes through this work, he will find that $k_{2}$ can take on only six different values, $-M / P$, $-M^{\prime} / P^{\prime},-M^{\prime \prime} / P^{\prime \prime},-N / Q,-N^{\prime} / Q^{\prime},-N^{\prime \prime} / Q^{\prime \prime}$, where $M, N, P, Q$ have the values 


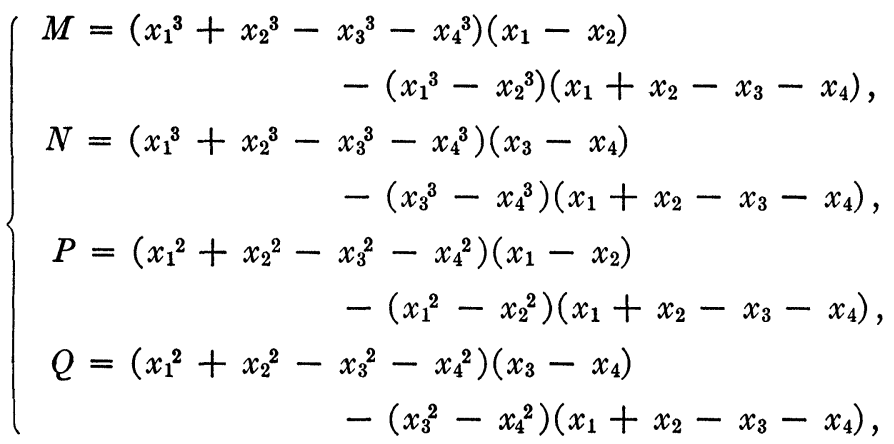

where $M^{\prime}, N^{\prime}, P^{\prime}, Q^{\prime}$ are obtained from $M, N, P, Q$ by interchange of the subscripts 2 and 3, and where $M^{\prime \prime}, N^{\prime \prime}$, $P^{\prime \prime}, Q^{\prime \prime}$ are obtained from $M, N, P, Q$ by replacing the subscripts $2,3,4$ by $4,2,3$, respectively. We thus see that $k_{2}$ can depend only on an equation of the sixth degree; that is, the twelf th degree equation mentioned above must, in this case, reduce to one of the sixth degree.

We now wish to go further, and show that this sixth degree equation can be broken up into quadratic factors, whose coefficients are themselves roots of equations of degree not higher than the third. Consider the quadratic equation $k_{2}^{2}+t k_{2}+u=0$, whose roots are $-M / P$ and $-N / Q$, and whose left-hand member is therefore a priori a factor of the left-hand member of the sextic. We have

$$
t=\frac{M}{P}+\frac{N}{Q}=\frac{M Q+N P}{P Q}, \quad u=\frac{M N}{P Q} .
$$

It can now be verified that $t$ and $u$ each take on only three different values under the 24 permutations on the roots $x_{1}, x_{2}, x_{3}, x_{4}$. These values are

$$
\begin{aligned}
t_{1} & =\frac{M Q+N P}{P Q}, & u_{1} & =\frac{M N}{P Q}, \\
t_{2} & =\frac{M^{\prime} Q^{\prime}+N^{\prime} P^{\prime}}{P^{\prime} Q^{\prime}}, & u_{2} & =\frac{M^{\prime} N^{\prime}}{P^{\prime} Q^{\prime}}, \\
t_{3} & =\frac{M^{\prime \prime} Q^{\prime \prime}+N^{\prime \prime} P^{\prime \prime}}{P^{\prime \prime} Q^{\prime \prime}}, & u_{3} & =\frac{M^{\prime \prime} N^{\prime \prime}}{P^{\prime \prime} Q^{\prime \prime}} .
\end{aligned}
$$


The equation in $t$ having the roots $t_{1}, t_{2}, t_{3}$ has, moreover, coefficients which are rational functions of the coefficients of the given equation; this follows because the elementary symmetric functions of the $t$ 's are symmetric functions of the $x$ 's, as can again be verified easily. A similar statement can be made concerning the cubic equation in $u$ having the roots $u_{1}, u_{2}, u_{3}$.

The sextic in $k_{2}$ thus has a quadratic factor whose coefficients are roots of cubic equations; in fact it clearly has three such factors and may be written

$$
\left(k_{2}^{2}+t_{1} k_{2}+u_{1}\right)\left(k_{2}^{2}+t_{2} k_{2}+u_{2}\right)\left(k_{2}^{2}+t_{3}{ }_{2}+u_{3}\right)=0 .
$$

This completes, except for certain details which can be filled in easily enough, the a priori demonstration that the general quartic equation can be reduced to the form $y^{4}+c_{2} y^{2}=0$ without solving any equation of higher degree than the third. This demonstration does not, however, lead to a convenient determination of the necessary transformation.

3. The Transformation. The use of a special form of the Tschirnhaus transformation introduced by Hermite makes the computation of the transformed equation in $y$ relatively simple.* This depends on certain invariantive properties which need not be stated explicitly; I shall merely say that his transformation for the quartic is of the form

$$
\begin{aligned}
y=(a x+b) B+\left(a x^{2}+\right. & 4 b x+3 c) C \\
& +\left(a x^{3}+4 b x^{2}+6 c x+3 d\right) D,
\end{aligned}
$$

where $B, C$ and $D$ are the parameters, and where the original quartic is

$$
a x^{4}+4 b x^{3}+6 c x^{2}+4 d x+e=0 .
$$

Cayley $\dagger$ has computed the transformed quartic, as well as its invariants of the second and third degree. I make use of

* Comptes Rendus, vol. 46 (1858), p. 961; Oeuvres, vol. 2, Paris, 1908, pp. 30-37.

$\dagger$ Collected Papers, vol. 4, Cambridge, 1891, pp. 375-394; vol. 5, 1892, 449-453. 
these last two expressions. If we use $S$ and $T$ to represent the quadratic invariant $a e-4 b d+3 c^{2}$ and the cubic invariant $a c e+2 b c d-a d^{2}-e b^{2}-c^{3}$ of (11), and if we put

$$
\left\{\begin{array}{l}
\phi=a B^{2}+4 b B C+c\left(2 B D+4 C^{2}\right)+4 d C D+e D^{2}, \\
\Delta=\frac{2}{3}\left(B D-C^{2}\right),
\end{array}\right.
$$

then the corresponding invariants of the transformed equation, which we may denote by $S_{y}$ and $T_{y}$, are

$$
\left\{\begin{array}{l}
S_{y}=S \phi^{2}+18 T \phi \Delta+3 S^{2} \Delta^{2} \\
T_{y}=T \phi^{3}+S^{2} \phi^{2} \Delta+9 S T \phi \Delta^{2}+\left(54 T^{2}-S^{3}\right) \Delta^{3} .
\end{array}\right.
$$

I first show that the parameters $B, C$ and $D$ can be chosen so that the transformed equation will have $S_{y}{ }^{3}-27 T_{y}{ }^{2}$ equal to zero. This expression is clearly of the sixth degree in $\phi$ and $\Delta$, but it reduces to $\left(S^{3}-27 T^{2}\right)$ multiplied by the square of $\left(\phi^{3}-9 S \phi \Delta^{2}-54 T \Delta^{3}\right)$. Hence the condition $S_{y}^{3}-27 T_{y}^{2}=0$ is a cubic in $\phi / \Delta$ with known coefficients. If a root of this cubic is $r$, we have then to consider the equation $\phi=r \Delta$, which is a homogeneous, quadratic equation in $B$, $C$ and $D$. One of these can be taken equal to zero, and the equation solved for the ratio of the other two.

Now it is well known that any quartic for which $S^{3}-27 T^{2}$ is equal to zero can be reduced by a linear fractional transformation (which is reducible to a Tschirnhaus transformation) to the normal form $y^{4}+c_{2} y^{2}=0$. In fact it is not difficult to show that this reduction requires only the solution of quadratic equations, but I shall not reproduce the details of this calculation. Finally, since the product of two Tschirnhaus transformations is itself a Tschirnhaus transformation, we arrive at the desired result.

The UnIVERsity of Rochester 